\title{
Symbol Error Rate as a Function of the Residual ISI Obtained by Blind Adaptive Equalizers for the SIMO and Fractional Gaussian Noise Case
}

\author{
Monika Pinchas \\ Department of Electrical and Electronic Engineering, Ariel University, 40700 Ariel, Israel \\ Correspondence should be addressed to Monika Pinchas; monika.pinchas@gmail.com
}

Received 26 May 2013; Accepted 26 June 2013

Academic Editor: Ming Li

Copyright (C) 2013 Monika Pinchas. This is an open access article distributed under the Creative Commons Attribution License, which permits unrestricted use, distribution, and reproduction in any medium, provided the original work is properly cited.

\begin{abstract}
A nonzero residual intersymbol interference (ISI) causes the symbol error rate (SER) to increase where the achievable SER may not answer any more on the system's requirements. In the literature, we may find for the single-input-single-output (SISO) case a closed-form approximated expression for the SER that takes into account the achievable performance of the chosen blind adaptive equalizer from the residual ISI point of view and a closed-form approximated expression for the residual ISI valid for the singleinput-multiple-output (SIMO) case. Both expressions were obtained by assuming that the input noise is a white Gaussian process where the Hurst exponent $(H)$ is equal to 0.5. In this paper, we derive a closed-form approximated expression for the residual ISI obtained by blind adaptive equalizers for the SIMO case, valid for fractional Gaussian noise (fGn) input where the Hurst exponent is in the region of $0.5 \leq H<1$. Based on this new expression for the residual ISI, a closed-form approximated expression is obtained for the SER valid for the SIMO and fGn case. In this paper, we show via simulation results that the SER might get improved for increasing values of $H$.
\end{abstract}

\section{Introduction}

We consider a blind deconvolution problem in which we observe the multiple output of a finite impulse-response (FIR) single-input multiple-output (SIMO) channel from which we want to recover its input using adjustable linear filters (equalizers). In the field of communication, SIMO channels appear either when the signal is oversampled at the receiver or from the use of an array of antennas in the receiver [1-5]. It should be pointed out that for the SIMO case, the same information is transmitted through different subchannels, and all received sequences will be distinctly distorted versions of the same message, which accounts for a certain signal diversity [6]. Therefore, it is reasonable to assume that more information about the transmitted signal will be available at the receiver end [6]. SIMO transmission is widely replacing single-input-single-output (SISO) approach to enhance the performance via diversity combining [7]. It is well known that intersymbol interference (ISI) is a limiting factor in many communication environments where it causes an irreducible degradation of the bit error rate (BER) and symbol error rate (SER) thus imposing an upper limit on the data symbol rate [1]. In order to overcome the ISI problem, an equalizer is implemented in those systems. Recently [1], a closed-form approximated expression was derived for the achievable residual ISI obtained by blind adaptive equalizers in a SIMO system where the error that is fed into the adaptive mechanism which updates the equalizer's taps is expressed as a polynomial function of order three of the equalized output. But, this expression was obtained by assuming that the input noise is a white Gaussian process where the Hurst exponent $(H)$ is equal to 0.5 . A white Gaussian process is a special case $(H=0.5)$ of the fractional Gaussian noise (fGn) model [8]. FGn with $H \in(0.5,1)$ corresponds to the case of long-range dependency (LRD) [8]. As stated in [9], LRD implies heavytailed probability density functions, which in general imply more random; see [10-13]. This point of view was recently detailed by $[14,15]$. In the literature, we may also find a closed-form approximated expression for the SER valid for the SISO case [16] that takes into account the performance 
of the chosen blind adaptive equalizer from the residual ISI point of view. But, this expression is not valid for the SIMO case and is based again on the assumption that the input noise is a white Gaussian process where the Hurst exponent $(H)$ is equal to 0.5 . Up to now, there is no closed-form approximated expression for the SER valid for the SIMO and fGn input case where the Hurst exponent is in the region of $0.5 \leq H<1$ that takes into account the performance of the chosen blind adaptive equalizer from the residual ISI point of view. Thus, the system designer still has to carry out many simulations in order to find those system parameters such as the equalizer's tap length and step-size parameter that will lead the system to the required SER.

In this paper, we propose for the real and two independent quadrature carriers case, a closed-form approximated expression for the achievable residual ISI obtained by blind adaptive equalizers for the SIMO and fGn input case that depends on the step-size parameter, equalizer's tap length, input constellation statistics, channel power, and the number of receive antennas used in the SIMO system and on $H$. The new closed-form approximated expression is applicable for type of blind adaptive equalizers used in a SIMO FIR channel where the error that is fed into the adaptive mechanism which updates the equalizer's taps can be expressed as a polynomial function of order three of the equalized output. Based on this new expression for the residual ISI, a closedform approximated expression is obtained for the SER valid for the SIMO and fGn case. As already mentioned, fGn with $H \in(0.5,1)$ corresponds to the case of LRD. Thus, it could be thought that the SER might increase as the value for $H$ increases due to the noise dependency from the different receive paths. But, according to simulation results, improved SER performance is seen for higher values of $H$.

The paper is organized as follows. After having described the system under consideration in Section 2, the closed-form approximated expressions for the residual ISI and SER are introduced in Section 3. In Section 4, simulation results are presented, and the conclusion is given in Section 5.

\section{System Description}

The system under consideration, illustrated in Figure 1, is the same system described in [1] (except for the input noise issue which will be discussed later on in this paper). In this paper, we make the following assumptions.

(1) The source sequence $x[n]$ belongs to a real or two independent quadrature carriers case constellation input with variance $\sigma_{x}^{2}$, where $x_{r}[n]$ and $x_{i}[n]$ are the real and imaginary parts of $x[n]$, respectively.

(2) The unknown subchannel $h^{(m)}[n](m=1,2,3, \ldots, M$ where $M$ is the number of subchannels) is a possibly nonminimum phase linear time-invariant filter. There is no common zero among all the subchannels.

(3) Each equalizer $c^{(m)}[n](m=1,2,3, \ldots, M)$ is a tapdelay line.

(4) The noise $w^{(m)}[n](m=1,2,3, \ldots, M)$ consists of $w^{(m)}[n]=w_{r}^{(m)}[n]+j w_{i}^{(m)}[n]$, where $w_{r}^{(m)}[n]$ and $w_{i}^{(m)}[n]$ are the real and imaginary parts of $w^{(m)}[n]$, respectively, and $w_{r}^{(m)}[n]$ and $w_{i}^{(m)}[n]$ are independent. Both $w_{r}^{(m)}[n]$ and $w_{i}^{(m)}[n]$ are fractional Gaussian noises (fGn) with zero mean. For $m=k$, we have $E\left[w_{r}^{(m)}[n] w_{r}^{(k)}[\tilde{n}]\right]=\sigma_{w_{r}}^{2} \delta[n-\tilde{n}]$ and $E\left[w_{i}^{(m)}[n] w_{i}^{(k)}[\widetilde{n}]\right]=\sigma_{w_{i}}^{2} \delta[n-\widetilde{n}]$. For $m \neq k$, we have $E\left[w_{r}^{(m)}[n] w_{r}^{(k)}[\widetilde{n}]\right]=\left(\sigma_{w_{r}}^{2} / 2\right)\left[(|m-k|-1)^{2 H}-\right.$ $\left.2(|m-k|)^{2 H}+(|m-k|+1)^{2 H}\right] \delta[n-\tilde{n}]$ and $E\left[w_{i}^{(m)}[n] w_{i}^{(k)}[\widetilde{n}]\right]=\left(\sigma_{w_{i}}^{2} / 2\right)\left[(|m-k|-1)^{2 H}-\right.$ $\left.2(|m-k|)^{2 H}+(|m-k|+1)^{2 H}\right] \delta[n-\tilde{n}]$, where $E[\cdot]$ denotes the expectation operator on $(\cdot), \delta$ is the Kronecker delta function, and $H$ is the Hurst exponent. In the following, we assume that $\sigma_{w_{i}}^{2}=\sigma_{w_{r}}^{2}$.

According to Figure 1, the $m$ th observation $y^{(m)}[n]$ $\left(y^{(m)}[n]=x[n] * h^{(m)}[n]+w^{(m)}[n]\right)$ is the result of a linear convolution between the source signal $x[n]$ and the corresponding channel response $h^{(m)}[n]$, corrupted by noise $w^{(m)}[n]$, where “*” denotes the convolution operation. The equalizer's output $z[n]$ is derived as follows:

$$
\begin{aligned}
z[n] & =\sum_{m=1}^{m=M} z^{(m)}[n]=\sum_{m=1}^{m=M} y^{(m)}[n] * c^{(m)}[n] \\
& =\sum_{m=1}^{m=M}\left(x[n] * h^{(m)}[n] * c^{(m)}[n]+w^{(m)}[n] * c^{(m)}[n]\right) \\
& =x[n]+p[n]+\widetilde{w}[n]
\end{aligned}
$$

where $p[n]$ is the convolutional noise $(p[n]=x[n] *$ $\xi[n]), \xi[n]=\sum_{m=1}^{m=M} h^{(m)}[n] * c^{(m)}[n]-\delta[n]$, and $\widetilde{w}[n]=$ $\sum_{m=1}^{m=M} w^{(m)}[n] * c^{(m)}[n]$. Next, we turn to the adaptation mechanism of the equalizer in each subchannel which is based on a predefined cost function $F[n]$ that characterizes the intersymbol interference; see, for example, [17-23]:

$$
\underline{c}^{(m)}[n+1]=\underline{c}^{(m)}[n]-\mu^{(m)} \frac{\partial F[n]}{\partial z[n]} \underline{y}^{(m)^{*}}[n],
$$

where $\mu^{(m)}$ is the step-size parameter in the subchannel, $c^{(m)}[n]$ is the equalizer vector, where the input vector is $y^{(m)}[n]=\left[y^{(m)}[n] \cdots y^{(m)}[n-N+1]\right]^{T}$, and $N$ is the equalizer's tap length. The operator $(\cdot)^{T}$ and $(\cdot)^{*}$ denote for transpose and conjugate of the function $(\cdot)$, respectively. Recently [1], a closed-form approximated expression was derived for the achievable residual ISI, valid for the SIMO case that depends on the step-size parameter, equalizer's tap length, input signal statistics, SNR, number of receive antennas, and channel power and is given in $(\mathrm{dB})$ units by [1]:

$$
\mathrm{ISI} \cong 10 \log _{10}\left(m_{p}\right)-10 \log _{10}\left(\sigma_{x_{r}}^{2}\right),
$$




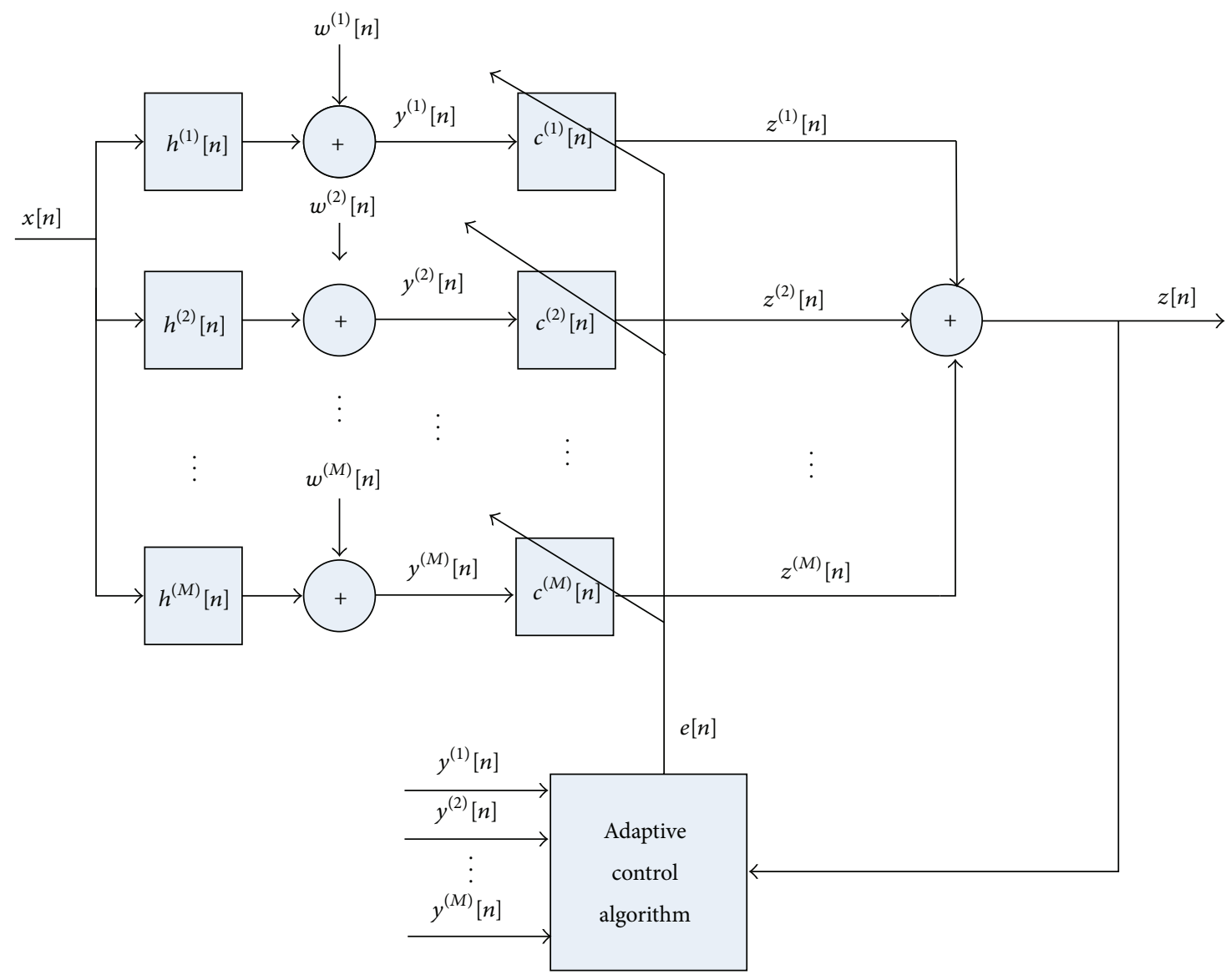

FIGURE 1: Block diagram of a baseband SIMO communication system.

where

$$
\begin{aligned}
& m_{p}=\min \left[\operatorname{Sol}_{1}^{m p_{1}}, \operatorname{Sol}_{2}^{m p_{1}}\right] \quad \text { for } \operatorname{Sol}_{1}^{m p_{1}}>0, \operatorname{Sol}_{2}^{m p_{1}}>0 \\
& \left.-2\left(a_{1}+3 \sigma_{x_{r}}^{2} a_{3}+\sigma_{x_{r}}^{2} a_{12}\right)\right) \\
& \text { or } m_{p}=\max \left[\operatorname{Sol}_{1}^{m p_{1}}, \operatorname{Sol}_{2}^{m p_{1}}\right] \text { for } \operatorname{Sol}_{1}^{m p_{1}} \cdot \operatorname{Sol}_{2}^{m p_{1}}<0 \text {, } \\
& +B\left(45 a_{3}^{2}+16 a_{3} a_{12}+9 a_{12}^{2}\right) \sigma_{\widetilde{w}_{r}}^{4} \\
& \text { where } \operatorname{Sol}_{1}^{m p_{1}}=\frac{-B_{1}+\sqrt{B_{1}^{2}-4 A_{1} C_{1} B}}{2 A_{1}} ; \\
& \operatorname{Sol}_{2}^{m p_{1}}=\frac{-B_{1}-\sqrt{B_{1}^{2}-4 A_{1} C_{1} B}}{2 A_{1}}, \\
& +\left(B \left(90 a_{3}^{2} \sigma_{x_{r}}^{2}+36 a_{3} a_{12} \sigma_{x_{r}}^{2}+12 a_{1} a_{3}+18 a_{12}^{2} \sigma_{x_{r}}^{2}\right.\right. \\
& \left.\left.+4 a_{1} a_{12}\right)-2 a_{12}-6 a_{3}\right) \sigma_{\widetilde{w}_{r}}^{2}, \\
& B=N \sigma_{x}^{2} \sum_{m=1}^{M} \mu^{m}\left(\sum_{k=0}^{k=R-1}\left|h_{k}^{(m)}[n]\right|^{2}+\frac{1}{\mathrm{SNR}}\right), \\
& A_{1}=\left(B\left(45 \sigma_{x_{r}}^{2} a_{3}^{2}+18 \sigma_{x_{r}}^{2} a_{3} a_{12}+6 a_{1} a_{3}+9 \sigma_{x_{r}}^{2} a_{12}^{2}+2 a_{1} a_{12}\right)\right. \\
& \left.-2\left(3 a_{3}+a_{12}\right)\right)+B\left(45 a_{3}^{2}+18 a_{3} a_{12}+9 a_{12}^{2}\right) \sigma_{\widetilde{w}_{r}}^{2}, \\
& C_{1}=\left(2\left(\sigma_{x_{r}}^{2}\right)^{2} a_{1} a_{12}+\sigma_{x_{r}}^{2} a_{1}^{2}+2 \mathbf{E}\left[x_{r}^{4}\right] \sigma_{x_{r}}^{2} a_{3} a_{12}\right. \\
& \left.+\mathbf{E}\left[x_{r}^{4}\right] \sigma_{x_{r}}^{2} a_{12}^{2}+2 \mathbf{E}\left[x_{r}^{4}\right] a_{1} a_{3}+\mathbf{E}\left[x_{r}^{6}\right] a_{3}^{2}\right) \\
& B_{1}=\left(B \left(12\left(\sigma_{x_{r}}^{2}\right)^{2} a_{3} a_{12}+6\left(\sigma_{x_{r}}^{2}\right)^{2} a_{12}^{2}+12 \sigma_{x_{r}}^{2} a_{1} a_{3}\right.\right. \\
& +\left(15 a_{3}^{2}+6 a_{3} a_{12}+3 a_{12}^{2}\right) \sigma_{\widetilde{w}_{r}}^{6} \\
& +4 \sigma_{x_{r}}^{2} a_{1} a_{12}+a_{1}^{2}+15 \mathrm{E}\left[x_{r}^{4}\right] a_{3}^{2} \\
& +\left(45 a_{3}^{2} \sigma_{x_{r}}^{2}+18 a_{3} a_{12} \sigma_{x_{r}}^{2}+6 a_{1} a_{3}+9 a_{12}^{2} \sigma_{x_{r}}^{2}+2 a_{1} a_{12}\right) \sigma_{\widetilde{w}_{r}}^{4} \\
& \left.+2 \mathbf{E}\left[x_{r}^{4}\right] a_{3} a_{12}+\mathbf{E}\left[x_{r}^{4}\right] a_{12}^{2}\right) \\
& +\left(a_{1}^{2}+12 a_{1} a_{3} \sigma_{x_{r}}^{2}+4 a_{1} a_{12} \sigma_{x_{r}}^{2}+15 a_{3}^{2} \mathbf{E}\left[x_{r}^{4}\right]\right.
\end{aligned}
$$




$$
\begin{aligned}
& +12 a_{3} a_{12}\left(\sigma_{x_{r}}^{2}\right)^{2}+2 a_{3} a_{12} \mathrm{E}\left[x_{r}^{4}\right] \\
& \left.+a_{12}^{2} \mathbf{E}\left[x_{r}^{4}\right]+6 a_{12}^{2}\left(\sigma_{x_{r}}^{2}\right)^{2}\right) \sigma_{\widetilde{w}_{r}}^{2},
\end{aligned}
$$

where $h_{k}^{(m)}[n]$ denotes the $k$ th tap of the $m$ th subchannel at time index $n, R$ is the subchannel length, and

$$
\sigma_{\widetilde{w}_{r}}^{2} \cong \sum_{m=1}^{m=M} \frac{\sigma_{x_{r}}^{2}}{M^{2} \operatorname{SNR} \sum_{k=0}^{k=R-1}\left|h_{k}^{(m)}[n]\right|^{2}}
$$

where SNR $=\sigma_{x}^{2} / \sigma_{w}^{2}$ and $a_{1}, a_{12}$, and $a_{3}$ are properties of the chosen equalizer and found by

$$
\begin{aligned}
& \Re\left(\frac{\partial F[n]}{\partial z[n]}\right) \\
& \quad=\left(a_{1}\left(z_{r}[n]\right)+a_{3}\left(z_{r}[n]\right)^{3}+a_{12}\left(z_{r}[n]\right)\left(z_{i}[n]\right)^{2}\right),
\end{aligned}
$$

where $\mathfrak{R}(\cdot)$ is the real part of $(\cdot)$ and $z_{r}, z_{i}$ are the real and imaginary parts of the equalized output $z[n]$, respectively.

As it was already implied earlier in this paper, the closedform approximated expression for the residual ISI [1] was obtained by assuming that the noise $w^{(m)}[n]$ is an additive Gaussian white noise $(H=0.5)$. Thus, it is not applicable for the fGn case (for $0.5 \leq H<1$ ). Therefore, in order to derive the expression for the SER applicable for the SIMO and fGn $(0.5 \leq H<1)$ case, a new expression for the achievable residual ISI is needed.

\section{Residual ISI and SER for the SIMO and Fractional Gaussian Noise Case}

In this section, a closed-form approximated expression is derived for the residual ISI valid for the SIMO and fGn case. Based on this new expression, the SER is obtained.

\subsection{Derivation of the Residual ISI}

Theorem 1. Consider the following assumptions.

(1) The convolutional noise $p[n]$ is a zero mean, white Gaussian process with variance $\sigma_{p}^{2}=E\left[p[n] p^{*}[n]\right]$. The real part of $p[n]$ is denoted as $p_{r}[n]$ and $E\left[p_{r}^{2}[n]\right]=$ $m_{p}$.

(2) The source signal $x[n]$ is a rectangular quadrature amplitude modulation (QAM) signal (where the real part of $x[n]$ is independent with the imaginary part of $x[n])$ with known variance and higher moments.

(3) The convolutional noise $p[n]$ and the source signal are independent.
(4) $\partial F[n] / \partial z[n]$ can be expressed as a polynomial function of the equalized output of order three.

(5) The gain between the source and equalized output signal is equal to one.

(6) The convolutional noise $p[n]$ is independent with $\widetilde{w}[n]$.

(7) The added noise is fGn as defined in the previous section in assumption (4).

(8) The Hurst exponent is in the range of $0.5 \leq H<1$.

The residual ISI expressed in ( $\mathrm{dB})$ units may be defined as (3), (4), (5), (6), (7), and (8), where SNR $=\sigma_{x}^{2} / \sigma_{w}^{2}, a_{1}, a_{12}, a_{3}$ are properties of the chosen equalizer and found by (10) and

$$
\sigma_{\widetilde{w}_{r}}^{2} \cong \sum_{m=1}^{M} \frac{\sigma_{x_{r}}^{2}}{M^{2} \operatorname{SNR} \sum_{k=0}^{k=R-1}\left|h_{k}^{(m)}[n]\right|^{2}}(1+H(2 H-1)(M-1)) .
$$

Comments. Please note that for $H=0.5$ (Gaussian white noise case), the expressions for $\sigma_{\widetilde{w}_{r}}^{2}$ given in (11) and (9) are equivalent. By repeating the steps in [1] for the calculation of the expression of the residual ISI the only place where the difference between the assumption of $w_{r}(n)$ and $w_{i}(n)$ being Gaussian white noises or fractional Gaussian noises has a major role on the total result of the approximated derived expression for the residual ISI, is in the calculation of $\sigma_{\widetilde{w}_{r}}^{2}$. Thus, we bring here only the various steps that led to (11).

It should be pointed out that assumptions (1)-(6) from above, are precisely the same assumptions made in [1].

Proof. The real part of $\widetilde{w}[n]$, namely, $\widetilde{w}_{r}[n]$ may be expressed as follows:

$$
\begin{aligned}
\widetilde{w}_{r} & {[n] } \\
= & \sum_{m=1}^{M} \sum_{k=0}^{k=N-1}\left(c_{r}^{(m)}[k] w_{r}^{(m)}[n-k]-c_{i}^{(m)}[k] w_{i}^{(m)}[n-k]\right),
\end{aligned}
$$

where $c_{r}^{(m)}[k]$ and $c_{i}^{(m)}[k]$ are the real and imaginary parts of $c^{(m)}[k]$, respectively. The variance of $\widetilde{w}_{r}[n]$ may be expressed by

$$
\begin{gathered}
\sigma_{\widetilde{w}_{r}}^{2}=E\left[\sum_{m=1}^{M} \sum_{k=0}^{k=N-1}\left(c_{r}^{(m)}[k] w_{r}^{(m)}[n-k]-c_{i}^{(m)}[k] w_{i}^{(m)}[n-k]\right)\right. \\
\cdot \sum_{p=1}^{M} \sum_{k k=0}^{k k=N-1}\left(c_{r}^{(p)}[k k] w_{r}^{(p)}[n-k k]\right. \\
\left.\left.-c_{i}^{(p)}[k k] w_{i}^{(p)}[n-k k]\right)\right]
\end{gathered}
$$


which can be also written as follows:

$$
\begin{aligned}
& \sigma_{\widetilde{w}_{r}}^{2}=\sum_{m=1}^{M} \sigma_{w_{r}}^{2} \sum_{k=0}^{k=N-1}\left|c^{(m)}[k]\right|^{2} \\
&+\sum_{m=1, m \neq p} \sum_{p=1, p \neq m}^{M} \sum_{k=0}^{k=N-1} E\left[c_{r}^{(m)}[k] c_{r}^{(p)}[k]\right. \\
& \cdot w_{r}^{(m)}[n-k] w_{r}^{(p)}[n-k] \\
&+c_{i}^{(m)}[k] c_{i}^{(p)}[k] \\
&\left.\cdot w_{i}^{(m)}[n-k] w_{i}^{(p)}[n-k]\right]
\end{aligned}
$$

or by

$$
\begin{aligned}
\sigma_{\widetilde{w}_{r}}^{2}= & \sum_{m=1}^{M} \sigma_{w_{r}}^{2} \sum_{k=0}^{k=N-1}\left|c^{(m)}[k]\right|^{2} \\
+ & \sum_{m=1, m \neq p}^{M} \sum_{p=1, p \neq m}^{M} \frac{\sigma_{w_{r}}^{2}}{2}\left[(|m-p|-1)^{2 H}-2(|m-p|)^{2 H}\right. \\
& \left.+(|m-p|+1)^{2 H}\right] \\
& \cdot \sum_{k=0}^{k=N-1}\left[c_{r}^{(m)}[k] c_{r}^{(p)}[k]+c_{i}^{(m)}[k] c_{i}^{(p)}[k]\right] .
\end{aligned}
$$

According to [24],

$$
\begin{aligned}
& 0.5\left[(|m-p|-1)^{2 H}-2(|m-p|)^{2 H}+(|m-p|+1)^{2 H}\right] \\
& \simeq H(2 H-1)|m-p|^{2 H-2} .
\end{aligned}
$$

Thus, substituting (16) into (15) yields to

$$
\begin{aligned}
\sigma_{\widetilde{w}_{r}}^{2} \cong & \sum_{m=1}^{M} \sigma_{w_{r}}^{2} \sum_{k=0}^{k=N-1}\left|c^{(m)}[k]\right|^{2} \\
& +\sigma_{w_{r}}^{2} H(2 H-1) \sum_{m=1, m \neq p}^{M} \sum_{p=1, p \neq m}^{M}|m-p|^{2 H-2} \\
& \times \sum_{k=0}^{k=N-1}\left[c_{r}^{(m)}[k] c_{r}^{(p)}[k]+c_{i}^{(m)}[k] c_{i}^{(p)}[k]\right] .
\end{aligned}
$$

This expression (17) can be upper limited by

$$
\begin{aligned}
& \sigma_{\widetilde{w}_{r}}^{2} \leq \sum_{m=1}^{M} \sigma_{w_{r}}^{2} \sum_{k=0}^{k=N-1}\left|c^{(m)}[k]\right|^{2} \\
&+\sigma_{w_{r}}^{2} H(2 H-1) \max _{p \neq m, p, m=1: M}\left[|m-p|^{2 H-2}\right] \\
& \times \mid \sum_{m=1, m \neq p}^{M} \sum_{p=1, p \neq m}^{M} \sum_{k=0}^{k=N-1}\left[c_{r}^{(m)}[k] c_{r}^{(p)}[k]\right. \\
&\left.+c_{i}^{(m)}[k] c_{i}^{(p)}[k]\right] \mid .
\end{aligned}
$$

According to the triangle inequality [25], we have

$$
\begin{aligned}
& \left|\sum_{m=1, m \neq p}^{M} \sum_{p=1, p \neq m}^{M} \sum_{k=0}^{k=N-1}\left[c_{r}^{(m)}[k] c_{r}^{(p)}[k]+c_{i}^{(m)}[k] c_{i}^{(p)}[k]\right]\right| \\
& \leq\left|\sum_{m=1, m \neq p}^{M} \sum_{p=1, p \neq m}^{M} \sum_{k=0}^{k=N-1}\left[c_{r}^{(m)}[k] c_{r}^{(p)}[k]\right]\right| \\
& +\left|\sum_{m=1, m \neq p}^{M} \sum_{p=1, p \neq m}^{M} \sum_{k=0}^{k=N-1}\left[c_{i}^{(m)}[k] c_{i}^{(p)}[k]\right]\right|
\end{aligned}
$$

According to the holder inequality [25], we may write

$$
\begin{aligned}
& \left|\sum_{m=1, m \neq p}^{M} \sum_{p=1, p \neq m}^{M} c_{r}^{(m)}[k] c_{r}^{(p)}[k]\right| \leq(M-1) \sum_{m=1}^{M}\left(c_{r}^{(m)}[k]\right)^{2}, \\
& \left|\sum_{m=1, m \neq p}^{M} \sum_{p=1, p \neq m}^{M} c_{i}^{(m)}[k] c_{i}^{(p)}[k]\right| \leq(M-1) \sum_{m=1}^{M}\left(c_{i}^{(m)}[k]\right)^{2} .
\end{aligned}
$$

By taking into account that $\max _{p \neq m, p, m=1: M}|m-p|^{2 H-2}=1$ for $0.5 \leq H<1$, we may write with the help of (18), (19), and (20) as follows:

$$
\sigma_{\widetilde{w}_{r}}^{2} \leq \sum_{m=1}^{M} \sigma_{w_{r}}^{2} \sum_{k=0}^{k=N-1}\left|c^{(m)}[k]\right|^{2}(1+H(2 H-1)(M-1)) .
$$

As already mentioned in the system description section, $\sum_{m=1}^{m=M} h^{(m)}[n] * c^{(m)}[n]=\xi[n]+\delta[n]$, where $\xi[n]$ stands for the error not having perfect equalization. Therefore, the expression $h^{(m)}[n] * c^{(m)}[n]$ could have been written as $h^{(m)}[n] * c^{(m)}[n]=\delta[n] / M+\widetilde{\xi}^{(m)}[n]$, where $\xi[n]=$ $\sum_{m=1}^{m=M} \widetilde{\xi}^{(m)}[n]$. Thus, for the case where the variance of $\widetilde{\xi}^{(m)}[n]$ can be considered as very small we may write

$$
\sum_{k=0}^{k=N-1}\left|c^{(m)}[k]\right|^{2} \cong \frac{1}{M^{2} \sum_{k=0}^{k=R-1}\left|h_{k}^{(m)}[n]\right|^{2}}
$$

Now, substituting (22) into (21) yields to

$$
\sigma_{\widetilde{w}_{r}}^{2} \leq \sum_{m=1}^{M} \frac{\sigma_{x_{r}}^{2}}{M^{2} \operatorname{SNR} \sum_{k=0}^{k=R-1}\left|h_{k}^{(m)}[n]\right|^{2}}(1+H(2 H-1)(M-1)) .
$$

In practice, the variance of $\widetilde{\xi}^{(m)}[n]$ is not so small. Thus, the expression for $\sum_{k=0}^{k=N-1}\left|c^{(m)}[k]\right|^{2}$ is higher than that given in (22). Therefore, we may define $\sigma_{\widetilde{w}_{r}}^{2}$ as given in (11). This completes our proof.

3.2. Derivation of the SER for the SIMO Case. In this subsection, we derive the SER for a source signal $x[n]$ belonging to a rectangular QAM constellation, applicable for the SIMO and fGn case. 


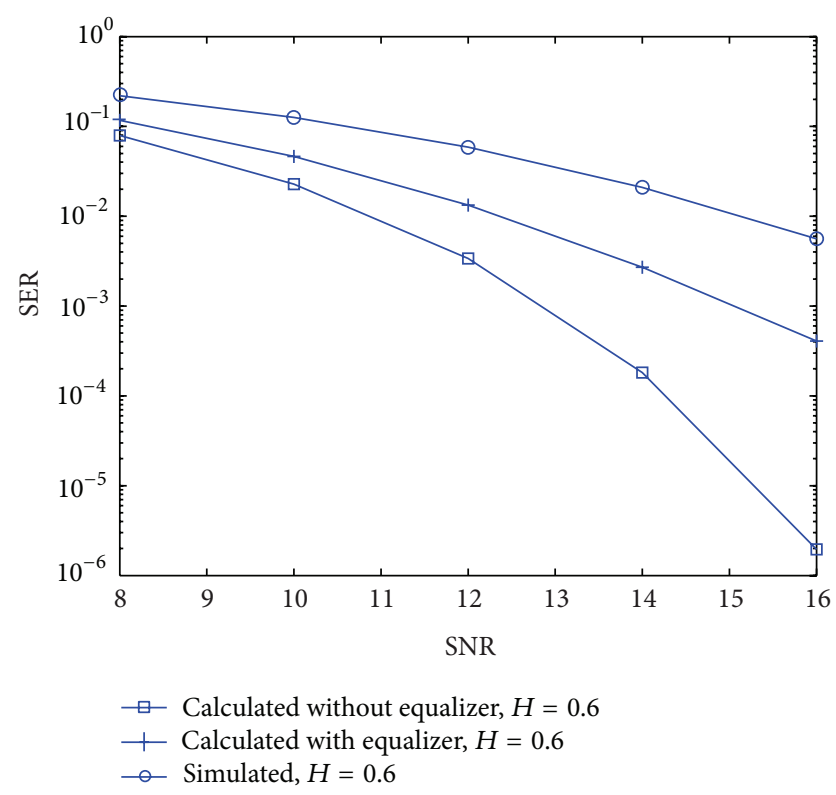

FIGURE 2: SER comparison with the following parameters: $d=1$, the step-size parameter $\mu=0.000005, N=17$, and $H=0.6$, channel case $\mathrm{A}$; the results were obtained for 1600000 symbols.

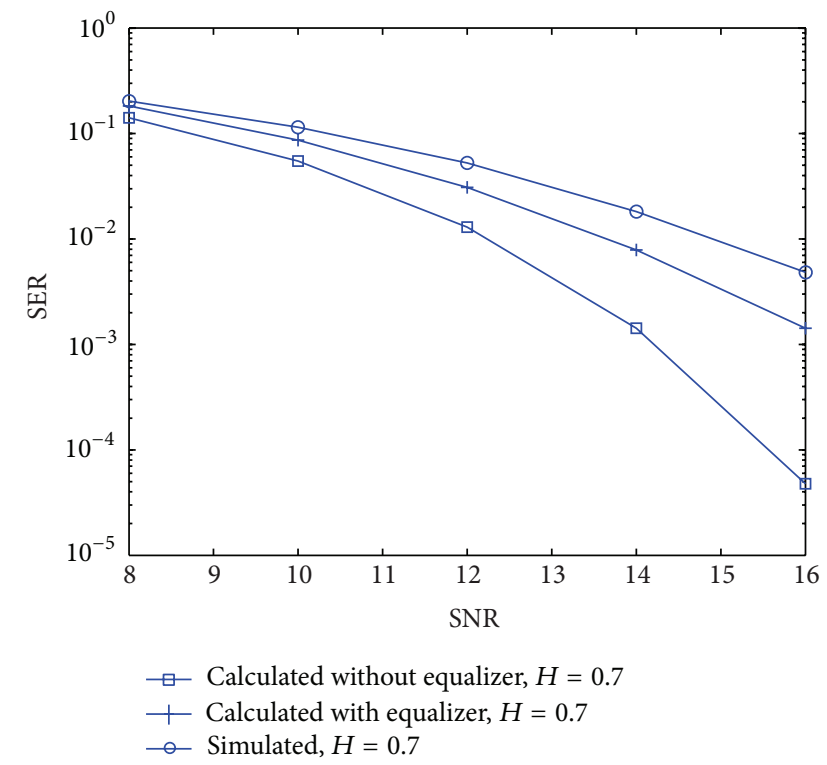

FIGURE 3: SER comparison with the following parameters: $d=1$, the step-size parameter $\mu=0.000005, N=17$, and $H=0.7$, channel case $\mathrm{A}$; the results were obtained for 1600000 symbols.

Theorem 2. Based on the assumptions given in the previous subsection, the SER for the SIMO and fGn case may be defined as follows:

$$
S E R_{\mathrm{QAM}}=4 \frac{\widetilde{M}-1}{\widetilde{M}} \mathrm{Q}\left(\frac{d}{\sigma_{T}}\right)\left(1-\frac{\widetilde{M}-1}{\widetilde{M}} \mathrm{Q}\left(\frac{d}{\sigma_{T}}\right)\right),
$$

where $\widetilde{M}=\sqrt{M_{\mathrm{QAM}}}$ and $M_{\mathrm{QAM}}$ is the number of signal points for a $M_{\mathrm{QAM}}$-ary $\mathrm{QAM}$ constellation, $d$ is half the distance

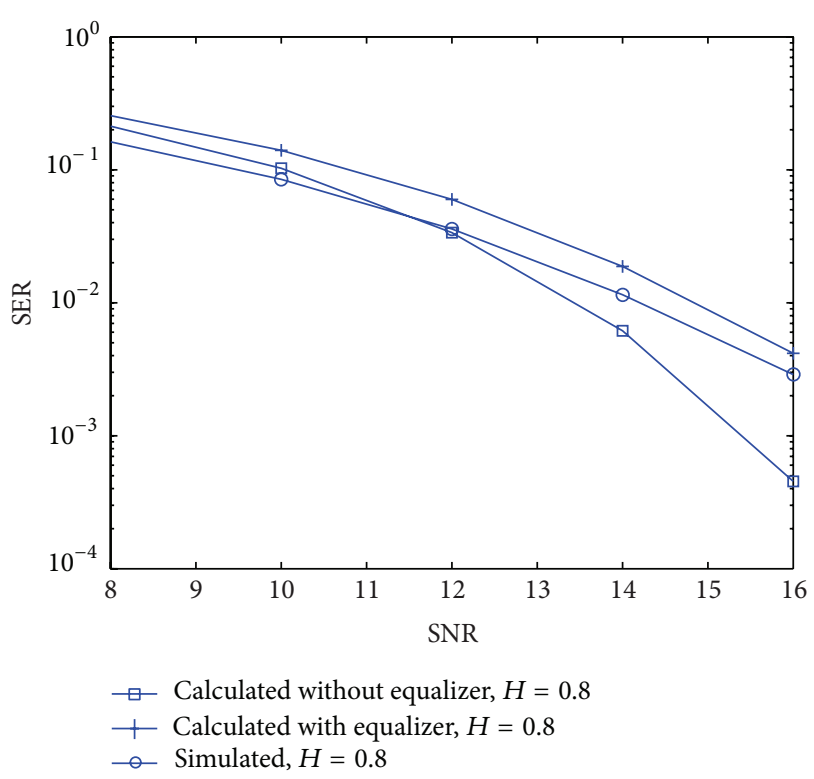

FIGURE 4: SER comparison with the following parameters: $d=1$, the step-size parameter $\mu=0.000005, N=17$, and $H=0.8$, channel case A; the results were obtained for 1600000 symbols.

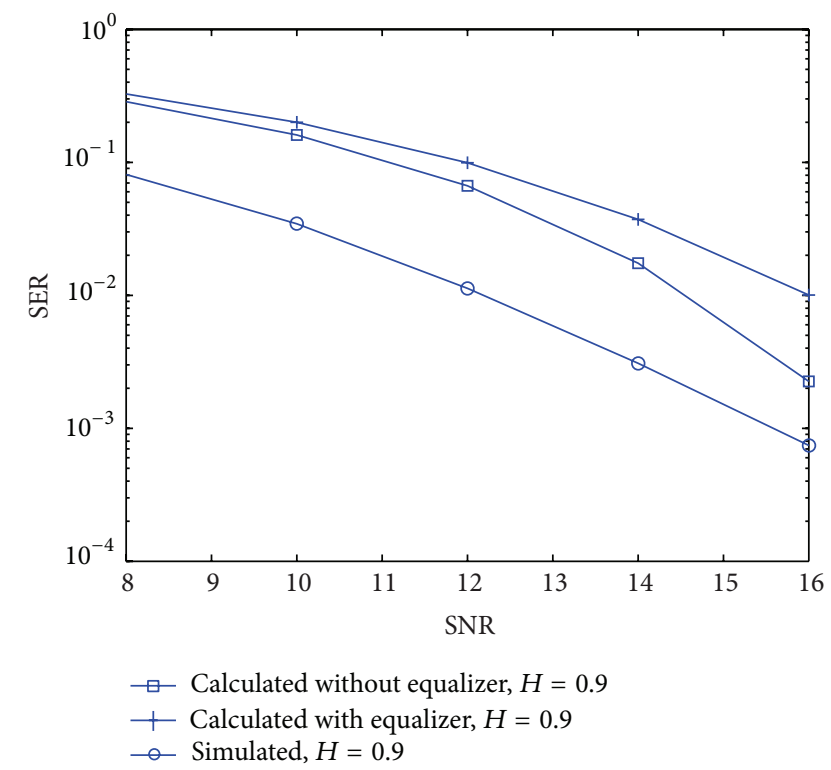

FIGURE 5: SER comparison with the following parameters: $d=1$, the step-size parameter $\mu=0.000005, N=17$, and $H=0.9$, channel case $\mathrm{A}$; the results were obtained for 1600000 symbols.

between adjacent $\sqrt{M_{\mathrm{QAM}}}$-ary pulse amplitude modulation (PAM) signals:

$$
\sigma_{T}=\sqrt{m_{p}+\sigma_{\widetilde{w}_{r}}^{2}} ; \quad Q\left(\frac{d}{\sigma_{T}}\right)=\frac{1}{\sqrt{2 \pi}} \int_{d / \sigma_{T}}^{\infty} e^{-u^{2} / 2} d u,
$$

and $m_{p}, \sigma_{\widetilde{w}_{r}}^{2}$ are according to (4) and (11), respectively. 


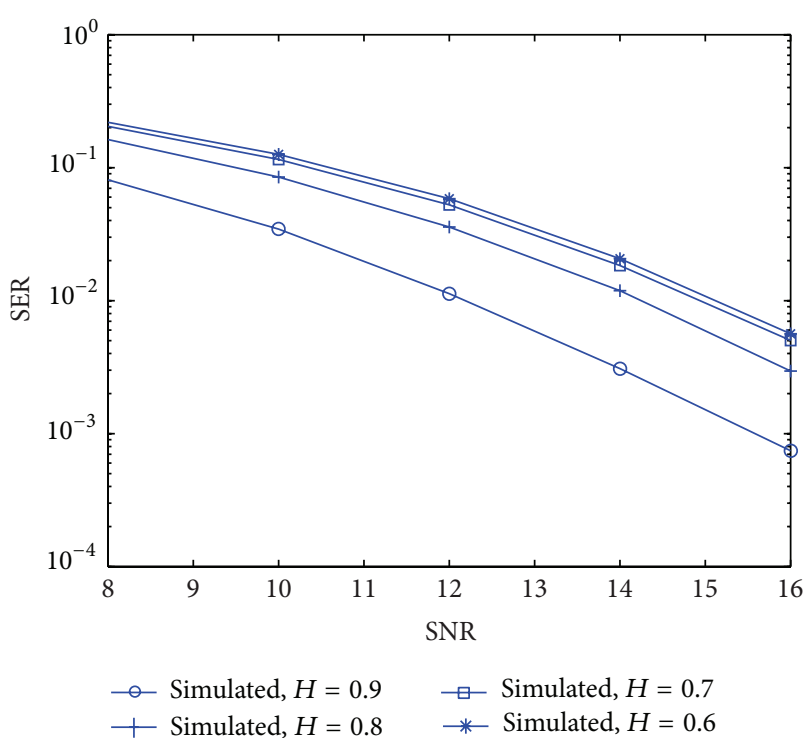

FIGURE 6: SER comparison with the following parameters: $d=1$, the step-size parameter $\mu=0.000005, N=17$, and $H=0.9,0.8,0.7,0.6$, channel case $\mathrm{A}$; the results were obtained for 1600000 symbols.

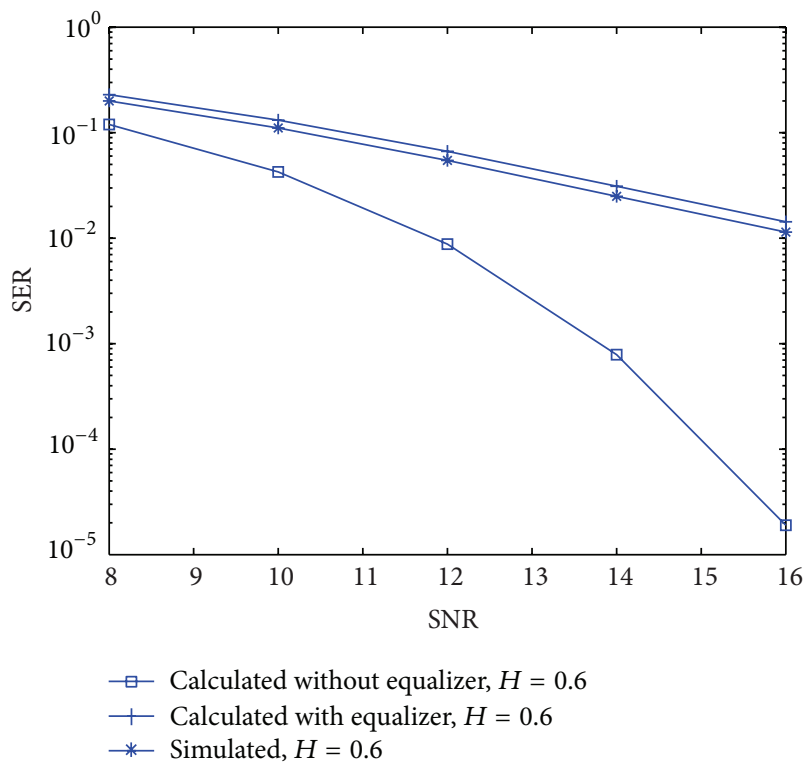

FIGURE 7: SER comparison with the following parameters: $d=1$, the step-size parameter $\mu=0.00002, N=13$, and $H=0.6$, channel case B; the results were obtained for 1600000 symbols.

Proof. According to (1), the equalized output is given by $z[n]=x[n]+p[n]+\widetilde{w}[n]$. The expression for the equalized output for the SISO and white Gaussian case (case one) looks quite similar to the expression for the equalized output for the SIMO and fGn case (case two). The difference between the two cases (case one and case two) lies in the fact that the variance of $p[n]$ and $\widetilde{w}[n]$ is very different. For each case, the expressions for the variance of the real part of $p[n]$ (named in this paper as $\left.m_{p}\right)$ and $\sigma_{\widetilde{w}_{r}}^{2}$ are different. Recently [16],

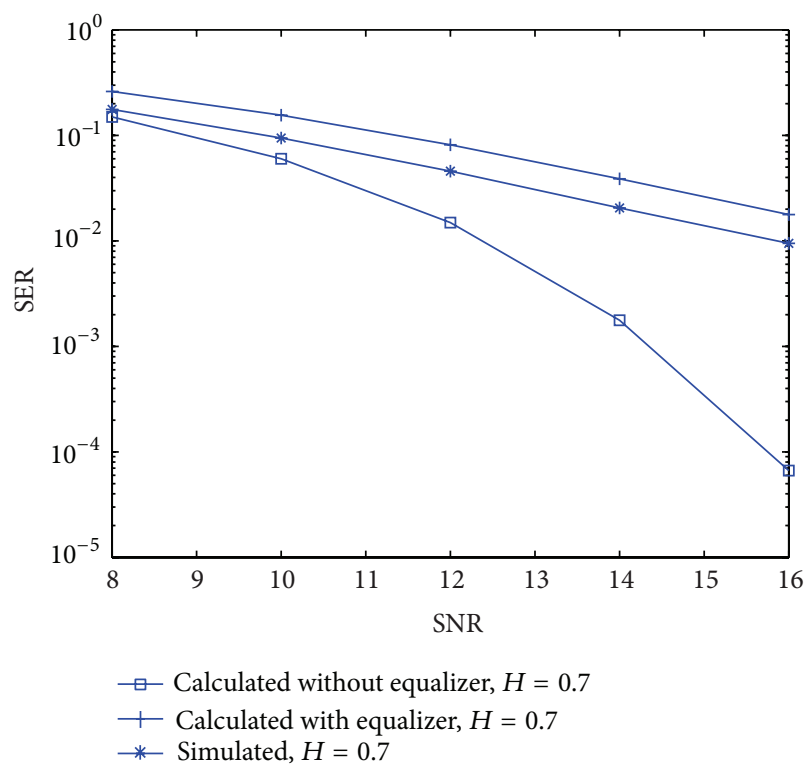

FIGURE 8: SER comparison with the following parameters: $d=1$, the step-size parameter $\mu=0.00002, N=13$, and $H=0.7$, channel case $\mathrm{B}$; the results were obtained for 1600000 symbols.

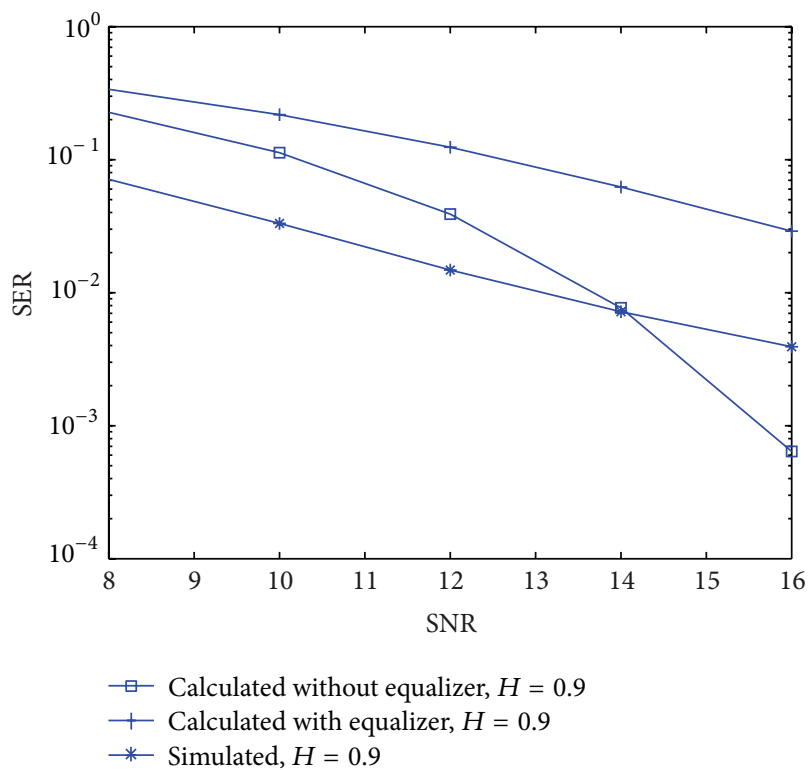

FIGURE 9: SER comparison with the following parameters: $d=1$, the step-size parameter $\mu=0.00002, N=13$, and $H=0.9$, channel case B; the results were obtained for 1600000 symbols.

a closed-form approximated expression was obtained for the SER, based on $z[n]=x[n]+p[n]+\widetilde{w}[n]$, valid for the SISO and white Gaussian $(H=0.5)$ case. The recently obtained expression for the SER [16] is recalled in (24), (25), where $m_{p}$ and $\sigma_{\widetilde{w}_{r}}^{2}$ were taken for the SISO and white Gaussian case. In order to obtain a closed-form approximated expression for the SER valid for the SIMO and fGn case, the recently obtained expression for the SER [16] was used with $m_{p}$ and 


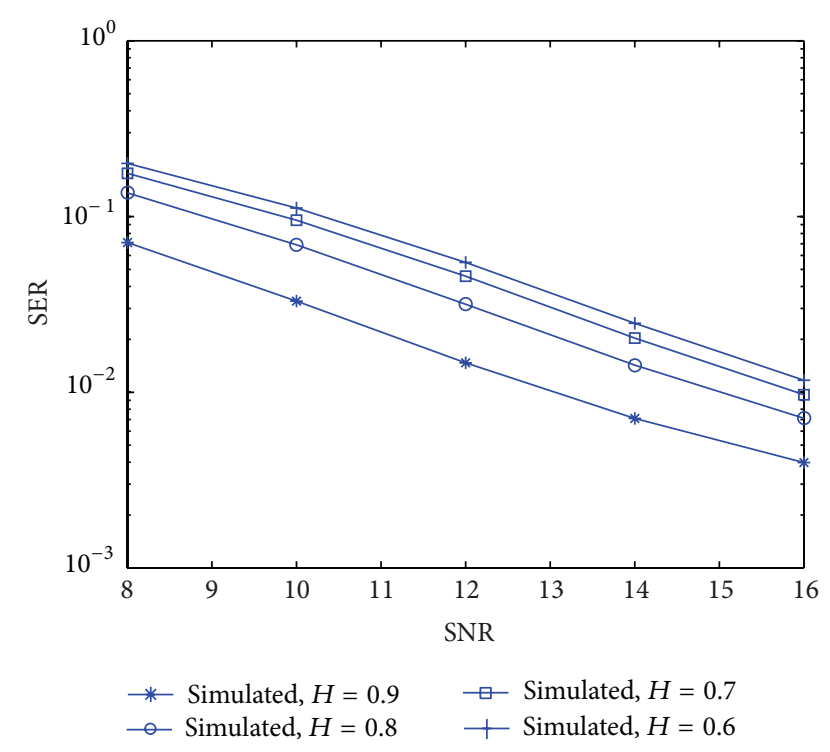

FIGURE 10: SER comparison with the following parameters: $d=$ 1 , the step-size parameter $\mu=0.00002, N=13$, and $H=$ $0.9,0.8,0.7,0.6$, channel case $\mathrm{B}$; the results were obtained for 1600000 symbols.

$\sigma_{\widetilde{w}_{r}}^{2}$ valid for the SIMO and fGn case. This completes our proof.

\section{Simulation}

In this section, we test our new proposed expression for the SER for the 16 QAM case (a modulation using $\pm\{1,3\}$ levels for in-phase and quadrature components) with Godard's algorithm [17] for different values of SNR and equalizer's tap length and for two different channel cases. The equalizer taps for Godard's algorithm [17] were updated according to the following:

$$
\begin{aligned}
c_{m}[n+1]= & c_{m}[n]-\mu_{G}\left(|z[n]|^{2}-\frac{E\left[|x[n]|^{4}\right]}{E\left[|x[n]|^{2}\right]}\right) \\
& \times z[n] y^{*}[n-m],
\end{aligned}
$$

where $\mu_{G}$ is the step size. The values for $a_{1}, a_{12}$, and $a_{3}$ corresponding to Godards's [17] algorithm are given by

$$
a_{1}=-\frac{E\left[|x[n]|^{4}\right]}{E\left[|x[n]|^{2}\right]} ; \quad a_{12}=1 ; \quad a_{3}=1
$$

In the following, we consider two channel cases. Channel case $A$ which is a four-channel model with channel coefficients determined according to the following:

$$
\begin{gathered}
h_{1}=\left[\begin{array}{llllll}
-0.0318+j 0.2330 & 0.3128-j 0.3693 & -0.3609+j 0.3810 & 0.6490 & -0.1110+j 0.0396
\end{array}\right], \\
h_{2}=\left[\begin{array}{llllll}
0.3005-j 0.0247 & 0.6784 & 0.6248-j 0.1316 & 0.1282-j 0.1411 & -0.0590-j 0.0366
\end{array}\right], \\
h_{3}=\left[\begin{array}{lllll}
-0.1351-j 0.2061 & -0.1274+j 0.5876 & 0.6401 & -0.1818-j 0.3354 & 0.0871-j 0.1216
\end{array}\right], \\
h_{4}=\left[\begin{array}{lllll}
0.2835+j 0.0204 & 0.6799 & 0.5936+j 0.0986 & 0.1938+j 0.2101 & -0.0333+j 0.1095
\end{array}\right] .
\end{gathered}
$$

Channel case $B$ which is a two-channel model with channel coefficients determined according to the following:

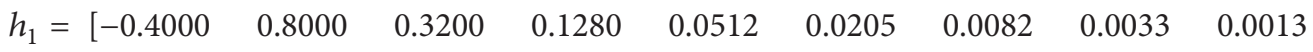

$$
\begin{aligned}
& \left.\begin{array}{llll}
0.0005 & 0.0002 & 0.0001 & 0.0000
\end{array}\right], \\
& h_{2}=\left[\begin{array}{lllllllllllll}
1.3000 & 0.6000-j 0.6364 & 0 & 0 & 0 & 0 & 0 & 0 & 0 & 0 & 0 & 0 & 0
\end{array}\right] \text {. }
\end{aligned}
$$

Please note that channel case B was also used in [1]. The equalizer was initialized by setting the center tap equal to one and all others to zero.

In the following, we denote the SER performance according to (24) as "calculated with equalizer." In addition, we wish to show the SER performance for the case where the residual ISI is not taken into account. Therefore, we denote in the following the SER performance that does not take into account the residual ISI as "calculated without equalizer."
Figures 2, 3, 4, and 5 and Figures 7, 8, and 9 show the SER performance for different values of $H$ as a function of SNR of our proposed expression (24) compared with the simulated results and with those calculated results that do not take into account the residual ISI. According to Figure 2 to Figure 5 and Figure 7 to Figure 9, a high correlation is observed between the simulated and calculated results (24), while the opposite is seen by comparing the simulated and those calculated results that do not take into account the 
residual ISI. Figures 6 and 10 show the SER performance for different values of $H$ in the range of $0.5<H<1$ as a function of SNR for channel cases A and B, respectively. It is reasonable to think that the SER might increase as the value for $H$ increases due to the noise dependency from the different receive paths. But, according to simulation results (Figures 6 and 10), improved SER performance is seen for higher values of $H$.

\section{Conclusion}

In this paper, we proposed for the real and two independent quadrature carrier cases, a closed-form approximated expression for the SER that takes into account the residual ISI obtained by blind adaptive equalizers and is applicable for the SIMO and fGn $(0.5 \leq H<1)$ input case. This new expression is applicable for the type of blind adaptive equalizers used in a SIMO FIR channel where the error that is fed into the adaptive mechanism which updates the equalizer's taps can be expressed as a polynomial function of order three of the equalized output. The expression for the SER depends on the system's parameters (step-size parameter, equalizer's tap length, input constellation statistics, channel power, and number of receive antennas used in the SIMO system and on $H$ ). Thus, there is no need anymore to carry out any simulation in order to find those system's parameters that will lead to the required SER performance. According to the simulation results, a high correlation is obtained between the calculated and simulated results for the SER. FGn with $H \in(0.5,1)$ corresponds to the case of LRD. Thus, it could be thought that the SER might increase as the value for $H$ increases due to the noise dependency from the different receive paths. But, according to the simulation results, improved SER performance is seen for higher values of $H$.

\section{References}

[1] M. Pinchas, "A closed-form approximated expression for the achievable residual ISI obtained by blind adaptive equalizers in a SIMO FIR channel," in Proceedings of the IEEE 27th Convention of Electrical and Electronics Engineers in Israel, Hilton Queen of Sheba, Eilat, Israel, November 2012.

[2] I. Moazzen, A. M. Doost-Hoseini, and M. J. Omidi, "A novel blind frequency domain equalizer for SIMO systems," in Proceedings of the International Conference on Wireless Communications and Signal Processing (WCSP '09), pp. 1-6, Nanjing, China, November 2009.

[3] D. Peng, Y. Xiang, Z. Yi, and S. Yu, "CM-based blind equalization of time-varying SIMO-FIR channel with single pulsation estimation," IEEE Transactions on Vehicular Technology, vol. 60, no. 5, pp. 2410-2415, 2011.

[4] A. Coskun and I. Kale, "Blind multidimensional matched filtering techniques for single input multiple output communications," IEEE Transactions on Instrumentation and Measurement, vol. 59, no. 5, pp. 1056-1064, 2010.

[5] S. Van Vaerenbergh, J. Via, and I. Santamaria, "Blind identification of SIMO wiener systems based on kernel canonical correlation analysis," IEEE Transactions on Signal Processing, vol. 61, no. 9, pp. 2219-2230, 2013.
[6] http://books.google.co.il/books?id=bimBH2czOZ0C.

[7] S. Chen, A. Wolfgang, and L. Hanzo, "Constant modulus algorithm aided soft decision directed scheme for blind spacetime equalisation of SIMO channels," Signal Processing, vol. 87, no. 11, pp. 2587-2599, 2007.

[8] M. Li and W. Zhao, "Quantitatively investigating locally weak stationarity of modified multifractional Gaussian noise," Physica A, vol. 391, no. 24, pp. 6268-6278, 2012.

[9] M. Pinchas, "Residual ISI obtained by blind adaptive equalizers and fractional noise," Mathematical Problems in Engineering, vol. 2013, Article ID 972174, 11 pages, 2013.

[10] B. B. Mandelbrot, Multifractals and $1 / f$ noise, Springer, New York, NY, USA, 1999.

[11] B. B. Mandelbrot and J. R. Wallis, "Noah, Joseph, and operational hydrology," Water Resources Research, vol. 4, no. 5, pp. 909-918, 1968.

[12] R. J. Adler, E. Raisa Feldman, and M. S. Taqqu, Eds., A Practical Guide to Heavy Tails: Statistical Techniques and Applications, Birkhäuser, Boston, Mass, USA, 1998.

[13] A. Mura, M. S. Taqqu, and F. Mainardi, "Non-Markovian diffusion equations and processes: analysis and simulations," Physica A, vol. 387, no. 21, pp. 5033-5064, 2008.

[14] M. Li and W. Zhao, "On 1/f noise," Mathematical Problems in Engineering, vol. 2012, Article ID 673648, 23 pages, 2012.

[15] M. Li and W. Zhao, "Visiting power laws in cyber-physical networking systems," Mathematical Problems in Engineering, vol. 2012, Article ID 302786, 13 pages, 2012.

[16] M. Pinchas, "Symbol error rate as a function of the residual ISI obtained by blind adaptive equalizers," in Proceedings of the Pervasive and Embedded Computing and Communication Systems (PECCS '13), Barcelona, Spain, February 2013.

[17] D. N. Godard, "Self-recovering equalization and carrier tracking in two-dimensional data communication systems," IEEE Transactions on Communications Systems, vol. 28, no. 11, pp. 1867-1875, 1980.

[18] S. Haykin, "Blind deconvolution," in Adaptive Filter Theory, S. Haykin, Ed., Chapter 20, Prentice-Hall, Englewood Cliffs, NJ, USA, 1991.

[19] M. Pinchas, "A MSE optimized polynomial equalizer for 16QAM and 64QAM constellation," Signal, Image and Video Processing, vol. 5, no. 1, pp. 29-37, 2011.

[20] O. Shalvi and E. Weinstein, "New criteria for blind deconvolution of nonminimum phase systems (channels)," IEEE Transactions on Information Theory, vol. 36, no. 2, pp. 312-321, 1990.

[21] M. Pinchas, The Whole Story Behind Blind Adaptive Equalizers/ Blind Deconvolution, e-books Publications Department Bentham Science Publishers.

[22] M. Pinchas and B. Z. Bobrovsky, "A maximum entropy approach for blind deconvolution," Signal Processing, vol. 86, no. 10, pp. 2913-2931, 2006.

[23] M. Pinchas, "What are the analytical conditions for which a blind equalizer will loose the convergence state?" Signal, Image and Video Processing, vol. 6, no. 2, pp. 325-340, 2012.

[24] M. Li and W. Zhao, "On bandlimitedness and lag-limitedness of fractional Gaussian noise," Physica A, vol. 392, no. 9, pp. 19551961, 2013.

[25] M. R. Spiegel, Mathematical Handbook of Formulas and Tables, SCHAUM's Outline Series, McGRAW-HILL, New York, NY, USA, 1968. 


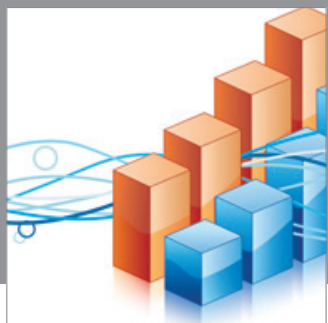

Advances in

Operations Research

mansans

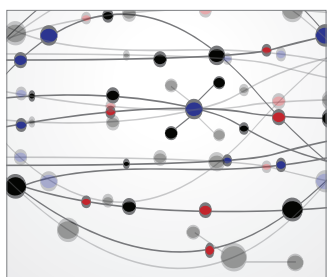

The Scientific World Journal
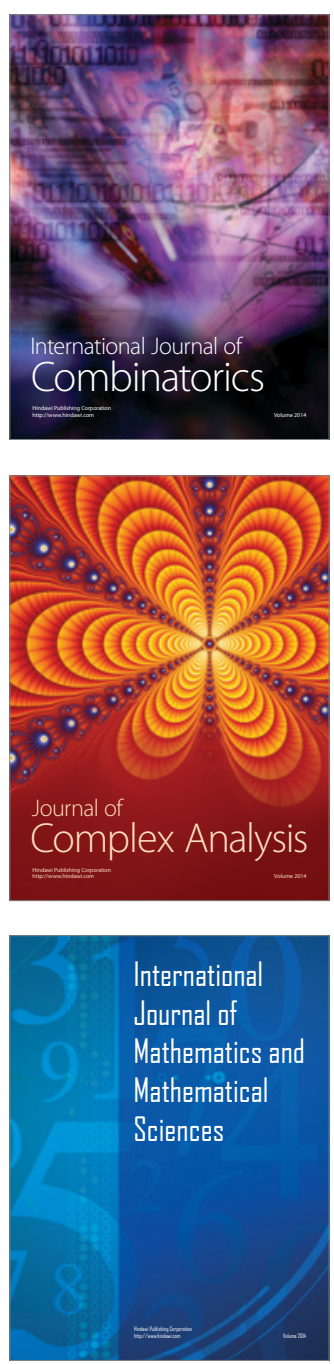
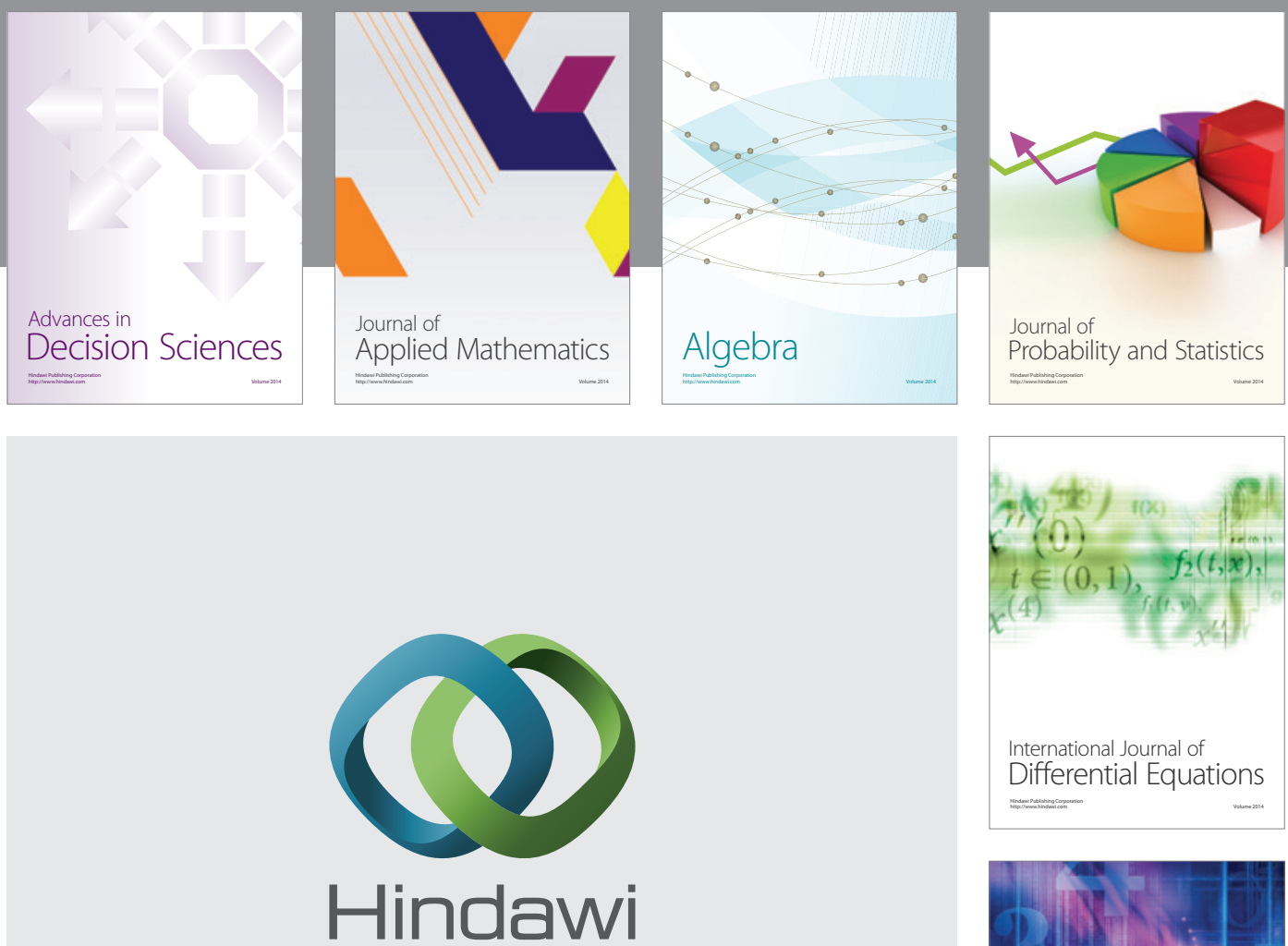

Submit your manuscripts at http://www.hindawi.com
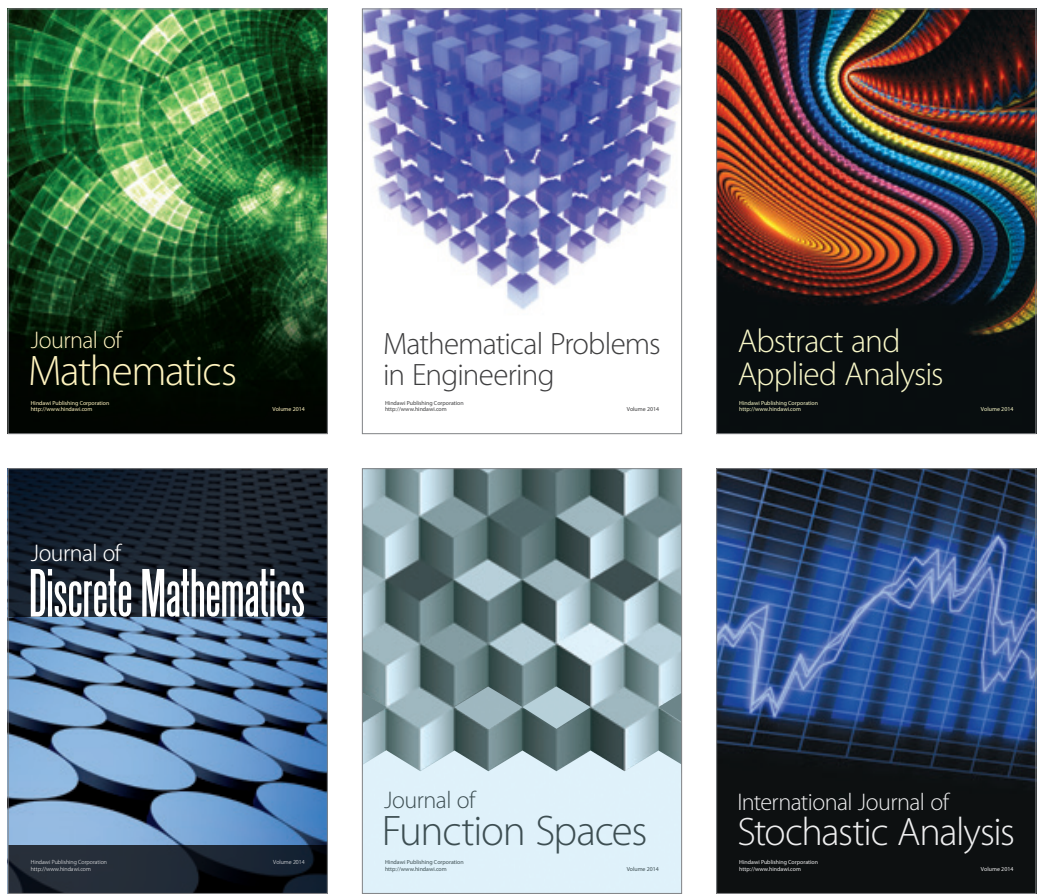

Journal of

Function Spaces

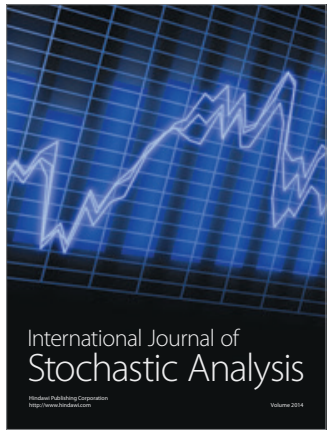

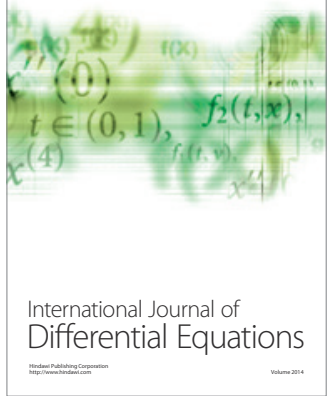
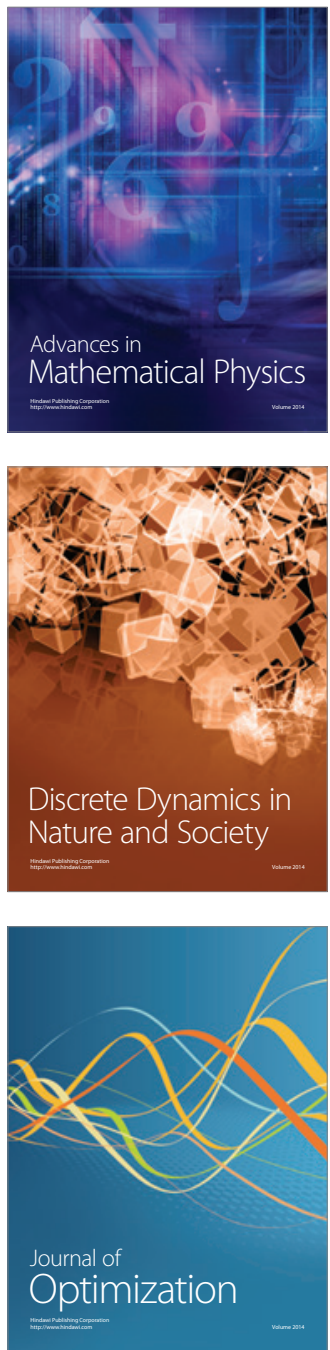